Revue européenne des sciences sociales

European Journal of Social Sciences

XLIV-134 | 2006

Quel(s) défi(s) pour les sciences sociales à l'heure de la mondialisation?

\title{
En hommage à Gérald Berthoud. Note sur la petite histoire du MAUSS (Mouvement anti-utilitariste en sciences sociales à l'usage des jeunes générations)
}

\section{Alain Caillé}

\section{OpenEdition}

\section{Journals}

Édition électronique

URL : http://journals.openedition.org/ress/282

DOI : $10.4000 /$ ress.282

ISSN : $1663-4446$

Éditeur

Librairie Droz

Édition imprimée

Date de publication : 1 juin 2006

Pagination : 45-58

ISBN : 9-782-600-01095-5

ISSN : 0048-8046

\section{Référence électronique}

Alain Caillé, "En hommage à Gérald Berthoud. Note sur la petite histoire du MAUSS (Mouvement antiutilitariste en sciences sociales à l'usage des jeunes générations) ", Revue européenne des sciences sociales [En ligne], XLIV-134 | 2006, mis en ligne le 14 octobre 2009, consulté le 05 mai 2019. URL: http://journals.openedition.org/ress/282 ; DOI : 10.4000/ress.282 
Alain CAILLÉ

\section{EN HOMMAGE À GÉRALD BERTHOUD NOTE SUR LA PETITE HISTOIRE DU MAUSS* (Mouvement anti-utilitariste en sciences sociales) à l'usage des jeunes générations}

\section{PROLOGUE}

J'ai déjà raconté ailleurs, et notamment dans Critique de la raison utilitaire (La Découverte, 1989, rééd. 2003), comment le MAUSS, le Mouvement antiutilitariste en sciences sociales, et la revue du même nom étaient nés, en 1981, à la suite d'une rencontre entre Berthoud et moi lors d'un colloque sur le don organisé au Centre Thomas More à l'Arbresle, où nous nous étions retrouvés l'un et l'autre étonnés et solidaires face au discours de la quasi-totalité des intervenants, économistes et psychanalystes pour la plupart, qui s'accordaient à considérer que le don ne peut être que de l'ordre d'une illusion dangereuse, à dissiper d'urgence pour faire apparaître la vérité profonde du sujet humain qui n'est qu'intérêt et calcul. Ce qui nous frappait, Berthoud et moi, c'était, en cette époque d'arrivée sur le devant de la scène de ce qu'on appelait alors les «nouveaux économistes »-autrement dit les champions idéologiques de ce qui sera désigné un peu plus tard comme le néo-libéralisme -, c'était la force du mouvement intellectuel qui consacrait à l'époque le triomphe du «modèle économique» dans les sciences sociales. Ou, si l'on préfère, de la Rational Action Theory ou, encore, de ce que j'appelle pour ma part l'axiomatique de l'intérêt.

Partout, en sociologie, en histoire, en biologie, en anthropologie, en philosophie morale et politique ou en psychanalyse, etc., on constatait que les explications légitimes se formulaient de plus en plus dans le langage de cette théorie économique généralisée que l'économiste Olivier Favereau, chef de file de l'Ecole dite des conventions, désigne sous le nom de théorie standard étendue. Dans chacun de ces champs il était de plus en plus raisonné comme si Marcel Mauss n'avait pas écrit l'Essai sur le don - ou comme si ce texte ne tirait nullement à conséquence - ou Polanyi La grande transformation. Et comme si, plus généralement, toute la sociologie classique, les grands textes de Marx, Tocqueville, Weber ou Durkheim étaient tout simplement nuls et non avenus.

C'est en vue de réagir contre cette évolution intellectuelle - dont nous ne mesurions d'ailleurs pas encore pleinement à l'époque toute la portée et dont nous ne soupçonnions pas qu'elle allait servir de justification avant la lettre au triomphe universel du marché et du capitalisme spéculatif sur toutes les autres formes d'organisation du rapport social -, que nous décidâmes, Berthoud et moi, de créer une sorte de bulletin de liaison entre chercheurs de diverses disciplines alarmés par la nouvelle donne intellectuelle et soucieux de ne pas laisser perdre l'héritage de la

\footnotetext{
* Pour faciliter la lecture et alléger la présentation, MAUSS est écrit dans ce texte sans les points.
} 
sociologie et de l'anthropologie classiques. Nos ambitions, on le voit, étaient grandes quant au fond, mais des plus modestes quant à la forme et aux moyens. Se borner à organiser la circulation de quelque chose comme des working papers nous suffisait tout à fait. Il faut croire cependant que nous n'avions pas trop mal évalué le caractère à la fois étrange et inquiétant de l'évolution de la division du travail intellectuel entre les diverses disciplines des sciences sociales et que nous n'étions pas absolument les seuls à nous en inquiéter puisque très vite, contre toute plausibilité, notre petit Bulletin rencontra un certain écho et rassembla suffisamment de lecteurs pour que l'aventure puisse continuer, se développer et prendre de l'ampleur. Aujourd'hui, vingt-cinq ans après, La Revue du MAUSS a en quelque sorte pignon sur rue. Elle est probablement la revue interdisciplinaire en sciences sociales la plus lue dans les pays francophones ${ }^{1}$ où elle a acquis, disons, de la légitimité et même une certaine respectabilité.

Tel n'a pas toujours été le cas. Longtemps les divers milieux académiques ou intellectuels institués se sont demandés ce qui se cachait derrière cette entreprise insolite: une revue qui ne s'autorisait que d'elle-même et qui, contrairement à toutes les règles et habitudes, ne bénéficiait de la protection ou de la recommandation d'aucune instance reconnue et donc d'aucune subvention, et ne dépendait de personne? Sans compter que nous intervenions dans divers champs disciplinaires sans y être autorisés en particulier dans aucun d'entre eux par les hiérarchies en place. Voilà qui était louche et qui autorisait tous les soupçons: soupçons d'amateurisme et de dilettantisme; soupçons de naïveté bien pensante (critiquer l'axiomatique de l'intérêt, cela pour beaucoup voulait nécessairement dire qu'on croyait que l'action pourrait s'expliquer au contraire par l'altruisme...); soupçons de visées idéologiques et politiques cachées. Si La Revue du MAUSS fait désormais pleinement partie du paysage intellectuel français, c'est parce que nos critiques se sont peu à peu convaincus que sous cette bannière il ne se cachait et ne se cache toujours rien d'autre que le souci d'organiser, selon le modèle légué par Marcel Mauss, un débat à la fois scientifique et éthico-politique qui tente d'expliciter et d'assumer ses propres présupposés tout en faisant droit à ceux des autres.

Je sais que Gérald Berthoud a souffert de ces soupçons. Ils ne s'expliquent pas seulement par la sottise ordinaire des spécialistes d'un seul champ et par la paresse intellectuelle si fréquente dans les milieux universitaires. C'est qu'il y avait en effet quelque chose de peu courant dans la démarche du MAUSS, qui surprenait et restait difficilement intelligible. Comment une telle revue a-t-elle pu fonctionner sans sembler a priori pouvoir rapporter à ses initiateurs la reconnaissance académique que seule procure désormais la publication dans des revues à comité de lecture et bien référencées par les organismes de notation? Et, plus prosaïquement encore, avec quel argent? Quelle force de travail? La question se pose d'autant plus que peu de revues nouvelles parviennent à s'implanter dans le secteur et que nombre de revues anciennes ne tiennent que sur leur vitesse acquise, grâce à des abonnements anciens et plus ou moins automatiquement renouvelés et à coup d'aides institutionnelles importantes.

Il m'a semblé qu'une des meilleures manières de rendre un amical hommage à Gérald Berthoud à l'occasion de son départ à la retraite était encore d'apporter des éléments de réponse à ces questions, des bribes d'informations susceptibles

\footnotetext{
1 Si on met à part le mensuel de vulgarisation Sciences Humaines.
} 
d'aider de plus jeunes à se lancer à leur tour dans ce type d'aventure. Comment le MAUSS a-t-il pu survivre et se développer? La clé du mystère est simple: grâce à l'anti-utilitarisme et à l'esprit du don déployé par ses protagonistes et sympathisants. Grâce aussi à beaucoup d'auto-exploitation. C'est cette petite histoire que je voudrais raconter ici, en actualisant et précisant une note que j'avais rédigée il y a trois ans en vue de procéder à un élargissement notable de l'association MAUSS pour permettre d'y accueillir notamment des jeunes chercheurs qui pouvaient légitimement se demander d'où venait et comment fonctionnait la revue avec laquelle ils sympathisaient et qu'ils s'apprêtaient maintenant à connaître de l'intérieur. La revue co-fondée par Gérald Berthoud. Voici donc, à quelques retouches et précisions près, ce que j'écrivais sur ce point il y a trois ans.

\section{UNE HISTOIRE TECHNIQUE DU MAUSS}

Il y a plusieurs années que je me suis promis à moi-même et ai promis à quelques autres de rédiger une petite note pour expliquer le fonctionnement du MAUSS à ceux qui n'en connaissent pas tous les arcanes et qui ignorent largement son histoire. Qu'est-ce que ce mouvement mystérieux, cette revue inclassable, ne relevant clairement d'aucune discipline déterminée et particulière ni d'aucune chapelle connue, auxquels le milieu intellectuel ou universitaire, interloqué, a longtemps et généreusement prêté des motivations peu claires voire franchement douteuses? Aujourd'hui que le MAUSS est enfin pris pour ce qu'il est un mouvement collectif informel qui agite des idées, dans le sillage de Marcel Mauss, à la croisée des sciences sociales et du champ politique -, et qu'il a accédé à une certaine respectabilité (au désespoir de certains Maussiens), une explication reste pourtant nécessaire puisqu'une belle jeunesse nous rejoint désormais, qu'elle se sent maussienne, qu'elle fait partie du MAUSS, mais se demande en même temps ce que cela signifie précisément, comment, sous quelle forme et en quels termes cette appartenance peut bien se traduire.

Inutile de revenir sur les débuts du MAUSS, présentés dans Critique de la raison utilitaire. Qu'il suffise de rappeler que le MAUSS s'est constitué bien classiquement (en 1981) sous la forme d'une association de 1901, avec une cotisation longtemps des plus modiques (40 FF) et que l'essentiel de son existence, outre l'organisation de quelques réunions, a été consacré à la publication du Bulletin puis de La Revue (trimestrielle) et enfin de La Revue semestrielle, à laquelle s'est adjointe une collection, la Bibliothèque du MAUSS ${ }^{2}$.

\section{Première période: Le Bulletin du MAUSS}

Trois grandes périodes, donc. Dans la première, celle du Bulletin, j'ai été président de l'association (et je le suis toujours depuis lors), le trésorier a été assez

\footnotetext{
2 Qu'il soit clair que le texte qui suit est une simple esquisse de l'histoire de la gestion et du fonctionnement technique du MAUSS, nullement son histoire intellectuelle. Dire qu'il s'agit d'une esquisse, c'est dire qu'elle est très certainement truffée d'erreurs ou d'imprécisions (je n'ai guère eu le temps de vérifier les dates et n'ai pu tabler que sur mes souvenirs) que les uns et les autres sont invités à rectifier en fonction de leurs propres souvenirs. Pour une rapide prise de connaissance de la revue, cf. www.revuedumauss.com
} 
longtemps Jérôme Lallement, le secrétaire général était au début Rigas Arvanitis (actuellement sociologue chercheur en Chine), remplacé ensuite par Cengiz Aktar (à moins que ce dernier ait remplacé Jérôme comme trésorier, je ne me rappelle plus très bien), Cengiz qui, ensuite, a longtemps travaillé au HCR (Afrique, Slovénie) et qui est maintenant en Turquie Monsieur Europe, celui qui pousse le plus à l'adhésion de la Turquie à l'Europe et qui y est considéré, me dit-on, comme la personne la plus qualifiée sur ce dossier. Comme quoi le MAUSS mène à tout (et pas nécessairement à condition d'en sortir...). Plus tard, Ahmet Insel est devenu secrétaire général (et l'est resté depuis lors) et Pierre Bitoun trésorier (ensuite relayé par Serge Latouche), Gérald Berthoud restant depuis le début jusqu'à aujourd'hui vice-président.

Autant pour les titres et fonctions officielles. L'important a été en fait le travail accompli pour préparer la revue et la diffuser chez quelques libraires. Depuis le début, c'est moi qui ai choisi ou commandé l'essentiel des textes publiés (je veux dire par là qu'il n'y a jamais eu de réunion formelle d'un comité de rédaction en bonne et due forme) après discussion avec les quelques uns qui étaient de l'aventure, Gérald Berthoud en premier lieu, Cengiz Aktar, Rigas Arvanitis ou Ahmet Insel, puis Serge Latouche très peu de temps après les tout débuts, me suggérant, isolément ou de concert, de publier tel ou tel texte. Le grand moment du MAUSS à l'époque était celui de la préparation matérielle du numéro, avec assemblage des textes, tentative de correction manuelle des fautes de frappe ou des pâtés les plus saillants, confection de la couverture grâce à des décalcomanies et au Lettraset, pagination à la main, etc. Elle se déroulait généralement chez Paulette Taieb, qui a disposé d'une des premières machines à écrire, une Olivetti, permettant de justifier un texte (alignement à droite) grâce à un petit écran sur lequel, merveille, on pouvait lire jusqu'à 5/6 mots à la fois et choisir entre deux ou trois polices de caractères grâce à un changement de «marguerite». Très vite, l'habitude fut prise par Paulette, durant ces soirées, de retaper mes présentations sur sa merveilleuse machine, ce qui permettait d'avoir au moins un texte justifié et bien lisible par numéro. Il faut le dire, ces séances de confection des numéros du Bulletin, qui réunissaient généralement Rigas, Cengiz, Ahmet et moi-même chez Paulette, et qui duraient jusqu'à une heure avancée de la nuit, ont été de purs moments de bonheur du travail fait en commun et de camaraderie pour lesquels je serai éternellement reconnaissant à ces premiers membres actifs du MAUSS ; des étrangers, tous (à l'exception de Paulette), cela mérite d'être noté, jeunes, et qui n'avaient rien à gagner à cette aventure mais n'ont jamais compté leur engagement ou demandé quoi que ce soit en échange. Simplement, ça leur plaisait.

L'autre partie du travail, moins drôle, consistait à remplir des dossiers pour obtenir de la commission paritaire de la presse des tarifs postaux avantageux, à rechercher une subvention du Centre national des Lettres (modeste subvention, que nous avons eue durant $2 / 3$ ans, à la fin du Bulletin et pendant une grande partie de la vie de la Revue, avant de la voir supprimée pour des raisons qui ne nous ont jamais été signifiées ${ }^{3}$ ), à expédier le bulletin à nos abonnés et à prospecter les libraires, piles de MAUSS à la main, pour qu'ils acceptent de nous prendre en

3 Nous avons récupéré cette subvention en 1996. Elle a d'abord été de 30'000 FF et est depuis deux ans de 5'000€ par an. 
dépôt. Dans ce travail, le charme de Cengiz, Ahmet et Rigas faisait merveille (surtout auprès des libraires femmes). Nous sûmes ainsi nouer des contacts réguliers avec une dizaine de librairies parisiennes qui allaient assurer l'essentiel de nos ventes. Incontestablement, notre plus belle réussite fut de nous mettre en bons termes avec M. Thorel qui dirigeait le premier étage de la librairie des PUF, consacré à la philosophie et aux sciences humaines, économiques et sociales. Très vite, M. Thorel (que nous remerciâmes ensuite par la délivrance d'un «tableau d'honneur du MAUSS ») décida de nous exposer en piles près de la caisse du premier étage, l'endroit le plus stratégique des PUF. C'est cette exposition miraculeuse qui nous valut d'être connus de nombreux universitaires étrangers de passage par Paris, à l'instar, par exemple, d'Axel Honneth (successeur d'Habermas à la tête de l'école de Francfort) qui me disait récemment qu'il avait eu presque tous les anciens Bulletins $d u$ MAUSS qui lui inspiraient la plus vive sympathie: ces couleurs gaies et dynamiques, le culot de publier une revue bourrée de fautes de frappe et dont la reliure avait une forte tendance à se détacher dès la première lecture, une revue qui ne faisait même pas semblant d'être un peu chic. Si nous n'avions pas procédé ainsi par un mélange d'inconscience et de nécessité faisant loi (ajoutées à un dilettantisme de bon aloi), on aurait presque pu croire rétrospectivement à un coup de génie publicitaire. Car, après, une fois le Bulletin devenu Revue du MAUSS aux Editions La Découverte, arborant une couverture sérieuse et grise (contre notre gré, bien évidemment et au nom de la science infaillible des services commerciaux), je ne sais pas si, comme l'a dit très vite Aldo Haesler ${ }^{4}$, le MAUSS «a perdu son âme» (je suis en fait bien persuadé du contraire), mais il a en tout cas perdu cette clientèle étrangère qui bien souvent, faute de le trouver encore près de la caisse des PUF, ne sut même pas que le bulletin avait survécu et prospéré sous une autre forme et sous d'autres couleurs.

\section{Deuxième période: La Revue du MAUSS}

Cette première période s'est achevée avec la proposition qui nous fut faite, à l'initiative de Thierry Paquot, alors directeur littéraire, de devenir une des revues de La Découverte (ex-Maspéro), avec Hérodote et après Critique de l'économie politique alors défunte. Il n'était guère imaginable de refuser une telle offre que rien n'avait laissé présager - je ne connaissais alors personne à La Découverte et qui permettait d'espérer une sérieuse augmentation des ventes par l'accès à des réseaux de diffusion nationaux et par le gain en respectabilité et en visibilité qu'il était possible d'escompter. Une sérieuse augmentation des ventes et donc la fin de nos difficultés financières chroniques et bien évidentes. Et puis, nous allions nous retrouver déchargés de tout le travail matériel, sympathique mais lassant passé un certain temps. La Découverte assurait tous les frais et tout le travail de la production et de la diffusion matérielles. Il n'y a en réalité pas grand chose à dire de cette période, sauf à discuter du fond de ce que nous avons alors produit, ce qui n'est pas mon propos ici, chacun pouvant s'en faire une idée par soi-même. Le travail

${ }^{4}$ Actuellement professeur de sociologie à l'Université de Caen. Longtemps, auparavant, professeur de sociologie et d'économie dans l'enseignement secondaire à Saint-Gall et chargé de cours, grâce à G. Berthoud, à l'Université de Lausanne. 
de préparation physique de la revue étant rendu inutile, les soirées trimestrielles chez Paulette Taieb disparurent. Cengiz avait trouvé un poste au Haut Commisariat pout les Réfugiés au cœur de l'Afrique; Rigas, qui avait aussi travaillé avec Bruno Latour et contribué à la naissance d'une autre revue informelle, latourienne cette fois, Pandore, était parti au Vénézuela comme sociologue des sciences. Restait Ahmet qui, pendant plusieurs années, fut le seul Maussien techniquement actif avec moi et Gérald Berthoud. Je n'aurais jamais pu fonctionner sans lui. Par la force des choses, recevant les suggestions des uns et des autres et les propositions de manuscrit qui nous étaient adressées, j'étais le seul à avoir une vision d'ensemble de la revue, mais je n'aurais jamais pu décider seul, ni matériellement ni, surtout, psychologiquement (puisque pour moi l'aventure n'avait et n'a encore de sens qu'à être aventure commune) des thèmes à mettre en avant, de la tonalité à donner à tel numéro, des articles à accepter ou refuser in fine. L'extraordinaire empathie et finesse d'Ahmet lui permettaient de juger au quart de tour, après que je lui eus expliqué ce qu'il y avait dans les divers articles possibles, de ce que nous devions publier ou non, faire ou ne pas faire, de ce qui était la ligne politique (au sens strict et large du terme à la fois) à suivre ou de celle qu'il fallait éviter. Avec Gérald, les longs coups de téléphone permettaient de pallier la distance et de mûrir peu à peu l'accord sur le projet proprement anthropologique et scientifique du MAUSS.

Je sens bien que les phrases que je viens d'écrire sont profondément trompeuses. Elles pourraient laisser croire que pendant tout ce temps là la revue a été faite par deux ou trois personnes. Rien ne serait plus faux. Elle a été d'abord la résultante de toutes les rencontres collectives ou interpersonnelles, formelles ou informelles, entre Maussiens divers. Outre les réunions publiques dont nous continuons la tradition, deux réunions à Peyriac-Minervois (près de Carcassonne) avec Ahmet, Serge, Gérald, Jean-Luc Boilleau et Pierre Lantz (la première fois) se sont essayées à fixer une doctrine du MAUSS. Celle que j'ai tenté d'exposer dans Critique de la raison utilitaire (1989), suite à une réunion de ce type notamment. Et il faudrait nommer tous ceux qui ont accompagné régulièrement l'aventure du MAUSS dans ces réunions diverses ou dans leurs articles. Les noms de Pascal Combemale, Yolande Bennarosh, Louis Baslé sont ceux qui me viennent spontanément à l'esprit. Auxquels il convient d'ajouter ceux de nos alliés girardiens: Jean-Pierre Dupuy nous prêtant des salles de l'Ecole polytechnique pour nos réunions, Lucien Scubla écrivant régulièrement dans le MAUSS et avec qui j'ai organisé diverses réunions MAUSS/CREA et, surtout, Mark Anspach, fidèle à ses deux inspirations, la girardienne et la maussienne (plus une troisième, la systémique batesonienne). Mais le travail de réalisation finale, la correction des manuscrits et des épreuves, le choix des orientations concrètes au numéro le numéro, tout cela en effet s'est fait largement à deux, Ahmet Insel et moi, sous la protection tutélaire de Gérald.

Au plan intellectuel, je crois que cette deuxième période a été réellement féconde. C'est à cette époque notamment que nous avons commencé à défricher l'histoire de l'utilitarisme, si inconnue alors et si importante pour nous. C'est dans cette période également que Jacques T. Godbout nous a rejoint en nous permettant de faire sortir la réflexion sur le don de son ghetto ethnologique pour lui donner toute sa portée sociologique et contemporaine. Au plan des ventes et de l'influence, le succès fut plus mitigé. Bizarrement, nous n'avons guère accru le 
nombre de nos abonnés. Comme Moïse ne pouvant jamais voir la terre promise ou Freud toujours empêché d'aller à Rome, nous n'avons jamais pu atteindre le seuil des 300 abonnés et restons scotchés, aujourd'hui encore, aux alentours de 270/280 abonnés payant, les abonnements institutionnels se substituant de plus en plus aux abonnés individuels ${ }^{5}$. Nous ne disposons donc pas du petit matelas financier qu'assure à d'autres (à nos amis de L'Homme et la société par exemple) un volant d'abonnés plus ancien et nettement plus substantiel. Tant mieux, en un sens. Voilà qui oblige à déployer quelque imagination pour trouver des thèmes susceptibles d'intéresser, numéro après numéro, en palliant la faiblesse des abonnements par de substantielles ventes en librairie. Mais celles-ci sont restées plutôt mesurées au temps de la revue trimestrielle (environ 600 à 700 par numéro). D'où une situation assez inconfortable à La Découverte (surtout après la suppression de la subvention du CNL) où l'on nous faisait comprendre que nous coûtions de l'argent et où, par ailleurs, François Gèze, le directeur - dont le soutien nous a toujours été infiniment précieux par ailleurs -, n'a jamais accepté à l'époque mes propositions de publier les livres des Maussiens que je lui suggérais. Tous les projets étaient jugés déficitaires et impraticables a priori. Seuls ont été publiés alors : les livres de Serge Latouche L'occidentalisation du monde et La planète des naufragés, jusqu'à ce que l'écart idéologique entre lui et La Découverte apparaisse trop grand'; L'esprit $d u$ don de Jacques T. Godbout (en collaboration avec moi, 1992) et, de moi, La Démission des clercs. La crise des sciences sociales et l' oubli du politique (1993). Avec l'accord des Maussiens de l'époque, j'ai donc préféré prendre les devants et reconquérir la liberté dont nous jouissions au temps du Bulletin, tout en nous donnant une chance de publier ceux de nos livres qui me/nous tenaient à cœur et notamment le livre de Philippe Rospabé, La dette de vie. Aux origines de la monnaie (1994), et celui de Jean-Luc Boilleau, Conflit et lien social. L'agôn contre la domination (1995). Par ailleurs, je commençais à fatiguer sérieusement de devoir produire un numéro tous les trois mois et aspirais à une périodicité moins astreignante, annuelle (j'ai pensé à L'année anti-utilitariste) ou semestrielle. C'est ainsi qu'a démarré la troisième phase du MAUSS.

\section{Troisième période: La Revue semestrielle et La Bibliothèque du MAUSS}

Pour mener à bien ces projets il nous fallait donc devenir éditeurs, non seulement d'une revue mais aussi de livres. Le pari que nous faisions était que rien ne nous empêchait de renouer avec la pratique du dépôt-vente en librairie, aidés en cela par l'augmentation du nombre des libraires qui nous connaissaient en France et par la proposition que nous voulions leur faire de leur laisser une commission très substantielle (40 à $45 \%$, je crois) sur chaque ouvrage vendu. Pour cela il fallait un petit capital de départ et changer les statuts du MAUSS. La barre fut placée assez haut : 5’000 francs français par associé. Dix-sept personnes se lancèrent ainsi dans l'aventure et devinrent les membres légaux de l'association MAUSS : Pierre Alphandéry, Mark Anspach, Gérald Berthoud, Pierre Bitoun,

5 Ce qui était vrai au moment de la première rédaction de cette note, fin 2002. Depuis lors, la fatalité a été vaincue et nous avons enfin dépassé ce seuil aussi fatidique que modeste.

$\checkmark$ Mais La Découverte a récemment publié une version de poche de L'occidentalisation du monde (2005). 
Jean-Luc Boilleau, Giovanni Busino, Alain Caillé, Pascal Combemale, Henri Denis, Anne-Marie Fixot, Jacques T. Godbout, Bernard Guerrien, Aldo Haesler, Ahmet Insel, Serge Latouche, Louis Moreau de Bellaing, Philippe Rospabé ${ }^{7}$ (G. Berthoud, A. Caillé et P. Combemale avançant en outre 5'000 FF chacun au MAUSS). Je pensais alors que nous aurions des réunions régulières pour décider du programme des publications et que nous nous retrouverions à plusieurs, comme au bon vieux temps, pour assumer les tâches matérielles. Il n'était même pas interdit d'espérer gagner un peu d'argent. Ph. Rospabé fit une étude prévisionnelle (d'ailleurs presque vérifiée ensuite) d'où il ressortait qu'il fallait tenir 2 ou 3 ans et qu'après nous pouvions espérer récupérer notre mise et même un peu audelà. Il était prêt quant à lui à s'investir systématiquement (et bénévolement) dans la préparation matérielle des numéros et des livres. Rien ne se passa pourtant comme prévu.

A la lettre que nous envoyâmes à 200 libraires pour leur faire part de nos intentions et des conditions mirifiques que nous leur consentions, il y eut très exactement une réponse (celle de Jérôme Grossmann de la librairie Tschann à Paris, je l'en remercie encore), qui eut le mérite de nous expliquer pourquoi nous faisions fausse route. Avec l'informatisation de la gestion, plus aucun libraire ne pouvait pratiquer, sauf exception, de système du dépôt-vente trop dérogatoire par rapport aux routines. Nous avons bien réussi à placer quelques dizaines d'exemplaires à droite ou à gauche, mais sans plus. Et de là à se faire payer effectivement... Il nous fallait au minimum trouver un diffuseur. C'est ainsi que nous nous tournâmes à nouveau ( je m'aperçois que je n'ai jamais utilisé autant de passés simples de toute ma vie que dans cette note...) vers La Découverte et aboutîmes à un accord de partenariat original, toujours en vigueur: en échange de l'usage du nom La Découverte et de l'accès à son réseau de diffusion et des conditions que lui font les imprimeurs, le MAUSS, qui règle absolument tous les frais (même si La Découverte avance la trésorerie), paie à La Découverte 8'000 francs forfaitaires (+2'000 FF pour la couverture) pour chaque titre et $12,5 \%$ par exemplaire vendu. De son côté, le MAUSS touche 36,5\% par exemplaire vendu (le reste allant à la distribution) et c'est avec ces rentrées (diminuées de 7\% versés à l'auteur au-delà de 1000 exemplaires vendus), ajoutées aux abonnements, qu'il doit financer toutes ses dépenses. Contrat en partie déséquilibré, peut-être (ainsi pour l'exercice 2001, La Découverte a perçu dans les 70’000 FF, alors que le MAUSS subissait un déficit de 44'000 FF), mais qui nous assure une grande liberté de manœuvre, l'accord de F. Gèze à nos propositions de publication étant désormais systématiquement acquis, sauf s'il nous venait en tête de publier des choses insensées. Précieuse liberté. Mais qui a ses exigences.

Au tout début, accord bizarre, il était entendu qu'un numéro sur deux de $L a$ Revue semestrielle serait produit par La Découverte et l'autre (les numéros pairs) par nous. C'est ainsi que le 1 et le 3 ressemblent à des livres de La Découverte et que le 2 ne ressemble à rien d'autre qu'à lui-même, la nouvelle formule n'allant trouver sa forme définitive qu'à partir du numéro 4. Nous en avons assuré la préparation, Ph. Rospabé et moi-même, aidés par mon épouse, Dominique. Prémices

Avec les adhésions ultérieures de Christian Cauvin, Philippe Chanial et François Fourquet, nous nous sommes finalement retrouvés à vingt. 
d'un changement notable par rapport aux plans initiaux. Du MAUSS, seul Philippe s'est alors investi dans les tâches matérielles qui s'annonçaient de plus en plus lourdes, P. Combemale de son côté assurant gracieusement la traduction d'un des chapitres du livre de Chantal Mouffe, Le politique et ses enjeux (1994). Les premiers livres produits, le Mouffe, le Caillé - Don, intérêt et désintéressement. Bourdieu, Mauss, Platon et quelques autres, 1994 (réédition augmentée 2005) avaient été composés avec l'aide des services de production de La Découverte avec un résultat catastrophique (mise en page minable, pas de nom d'auteur sur la tranche, etc.) - suite à notre inexpérience et aux rivalités entre services occasionnées par la création de la collection «Recherches»- et pour des coûts trop élevés pour le $\mathrm{n}^{\circ} 1$ et le $\mathrm{n}^{\circ} 3$ de La Revue.

Il allait donc falloir assurer nous-mêmes la préparation et la relecture des manuscrits. Philippe ne pouvait suffire à la tâche, à la fois parce qu'elle devenait très lourde et parce que nous ne pouvions pas nous voir fréquemment. Les réunions que je tentais d'organiser entre les associés du MAUSS pour discuter finances, programmation et problèmes techniques, furent un fiasco. La cuisine technique n'intéressait personne (à part Philippe et moi). Remarque: c'est tout à la gloire des Maussiens que ce souci de ne discuter que des problèmes de fond et pas des contingences matérielles. Dans la plupart des autres associations ou lieux de réunion divers, c'est rigoureusement le contraire qui prévaut très généralement.

C'est dans ce contexte que mon épouse, Dominique, me proposa de s'occuper de la gestion matérielle du MAUSS. Depuis des années, elle travaillait dans le marketing qualitatif, gagnant très correctement sa vie au prix de la rédaction d'une quinzaine de rapports (de 100 à 200 pages) par an et de la consommation de 4 paquets de cigarettes par 24 heures en période de stress, c'est-à-dire à peu près tout le temps. Je fus plus que réticent au début pour une foule de raisons, la principale étant que je reste nettement plus attaché au modèle de l'association des camarades et bons copains qu'à celui de la petite entreprise familiale. Mais, au bout du compte, il n'y avait guère le choix et, à l'usage, je dois attester que pour une activité comme la nôtre il n'y a pas d'autre modalité viable que celle de la petite entreprise familiale fonctionnant au nom et pour le compte de l'association des copains. Il serait trop long d'expliquer pourquoi en détail, car justement tout passe par une infinité de détails qui doivent, pour que les choses marchent, être réglés au jour le jour ou souvent à la minute, dans le sillage d'une préoccupation constante, et qui excluent toute possibilité d'une procédure lourde de consultation à distance et bien démocratique. On ne peut pas passer son temps à téléphoner, écrire ou emailer pour demander si tel mot est bien français, telle expression claire, telle traduction correcte, telle décision de tirage justifiée, tel abonnement bien enregistré, etc.

Juste une illustration chiffrée, pour se faire une idée de l'ampleur de la tâche. Nous avons publié en 2002, outre les deux numéros de La Revue du MAUSS, le livre de Denis Duclos, La société-monde, et celui de Laurent Geffroy, Garantir le revenu, avant l'été, et, après l'été, le livre de Stephen Kalberg, La sociologie historique comparative de Max Weber (250 pages) et celui de Christian Laval, L'ambition sociologique. Saint-Simon, Comte, Tocqueville, Marx, Durkheim, Weber (500 pages), soit plus de 1500 pages au format MAUSS ou au-delà (ajoutées aux 900 pages de la revue). Soit encore environ 5 millions de signes, l'équivalent de 20 à 25 romans de taille moyenne. Et, bien évidemment, il n'y a pas de 
photo quant au travail de préparation. Les textes que nous publions sont bourrés de notes et de références bibliographiques à vérifier, il leur arrive de jargonner et doivent donc être remis en français normalement intelligible. Quant aux traductions, il faut souvent les reprendre intégralement. D'autant plus, ajouterai-je, que Dominique a des exigences de clarté, de qualité et de rigueur que ma paresse naturelle m'interdit d'avoir spontanément. Il faut donc assurer tout ce travail, plus la gestion des abonnements, l'expédition des numéros et des commandes, la tenue de la comptabilité, etc. ${ }^{8}$

Au bout du compte, nous survivons, c'est déjà beaucoup, et avons publié les livres que nous voulions publier ainsi que d'autres réputés impubliables par des éditeurs «normaux »". Mais c'est un défi permanent. Au début, nous ne nous sommes tirés d'affaire que parce qu'inquiet de la situation, j'ai réussi une sorte de «coup», à l'instigation de Mark Anspach, me signalant qu'en Italie le livre de Jeremy Rifkin, La fin du travail, faisait un tabac. En plus, c'était de notre bord. J'ai donc pris contact avec Rifkin et ai tenté de le faire traduire à La Découverte. Devant le refus initial de F. Gèze, j'ai convaincu mon ami Christian Cauvin (professeur à HEC) d'avancer de l'argent pour faire une coédition avec La Découverte. Acceptation de Gèze. Malheureusement, un mois après, il préféra finalement publier le livre tout seul. C'est bien dommage. Nous aurions gagné dans l'affaire environ un million de francs. Malgré tout, il accorda au MAUSS une commission de $2 \%$ pour «apport d'affaire », ce qui rapporta environ 70 à 80'000 $\mathrm{FF}$, nous sauvant de la déroute financière.

Et maintenant? Reste à espérer, comme chaque année, que les numéros du MAUSS et les nouveaux livres publiés se vendent bien. La formule semestrielle nous vaut des protestations parce que les volumes sont trop gros et que personne n'a le temps de les lire in extenso (mais un numéro semestriel est à peine plus gros que deux trimestriels d'antan... Bon, disons, trois). Mais je trouve quant à moi la formule bonne. Elle nous permet de faire un réel travail de maturation sur chaque

8 Tout cela serait impossible sans cette belle auto-exploitation que seule permet la petite entreprise familiale. Pour la mener à bien, l'accord entre le MAUSS et la famille Caillé est le suivant. Le MAUSS contribue au loyer de l'appartement Caillé (à hauteur du quart de ce loyer), qui sert à la fois de bureau du MAUSS (trop petit) et d'entrepôt (cave pleine de numéros anciens ou en cours), il rembourse les frais courants (timbres) et achats de matériel (papier, gommes, crayons, photocopies, 2 vieux MAC achetés d'occasion et un demi PC qui fatigue), ainsi que la note d'une des deux lignes de téléphone qui fait aussi fax. Dominique, pour sa part, touchait jusqu'à il y a deux ans un peu moins (5'500 FF/mois) que le SMIC. Comme le MAUSS a été déficitaire au départ, son salaire ne lui a pas été versé pendant les quatre premières années (et n'a jamais fait l'objet d'un rattrapage). Il a fallu, depuis, face aux déficits chroniques du MAUSS, procéder avec elle à une rupture négociée de contrat pour raisons économiques. Elle ne travaille plus désormais pour le MAUSS que sous la forme de contrats à durée déterminée, au coup par coup, donc. A ce sujet, une petite précision ne sera peut-être pas inutile si j'en juge par la stupéfaction, il n'y a pas si longtemps, de notre ami Jacques Godbout, apprenant que nous n'avions aucune fortune personnelle et que nous n'étions pas propriétaires de notre appartement. Il lui semblait évident que, pour fonctionner comme nous le faisons, c'était que nous devions avoir de sérieux arriérés financiers. Eh bien, non: à part notre maison de Peyriac-Minervois, nous n'avons que des dettes et nous sommes donc, au moins sur ce plan, des anti-utilitaristes conséquents.

9 Par exemple, en 2004, le livre de Daniel Cefaï, L'enquête de terrain, ou, en 2005, le livre de Michel Terestchenko, Un si fragile vernis d'humanité. Banalité du mal, banalité du bien. Il se trouve que ces deux livres ont bien marché. 
sujet et donne aux lecteurs le sentiment d'avoir un vrai dossier entre les mains. Du coup, si nous piétinons en nombre d'abonnés, nous réalisons des ventes fort honorables en librairie, de l'ordre de 500 à 1500 selon les cas. Mais faute d'un nombre d'abonnés suffisants, nous restons toujours à la merci d'un échec et notamment de la mévente d'un numéro du MAUSS, puisque très gros ils coûtent très chers. C'est ainsi, par exemple, que les comptes 2001 ont été catastrophiques, en raison du four total et imprévu (mais rétrospectivement annonciateur d'autres fours, électoraux ceux-là...) du n 16 sur L'autre socialisme, comme si le mot était désormais répulsif. Et si le $\mathrm{n}^{\circ} 18$, Travailler est-il (bien) naturel?, a été épuisé très vite comme La Découverte nous l'a annoncé, me laissant espérer un triomphe, c'est parce que, comme souvent, ils n'ont pas respecté nos consignes et l'ont tiré à 1200 exemplaires au lieu des 2000 demandés. D'où un manque à gagner considérable.

Ici aussi, je m'aperçois que cet exposé du fonctionnement de La Revue du MAUSS semestrielle est totalement biaisé et trompeur. Soucieux de ne rien cacher des modalités concrètes de sa production et de sa gestion et de faire comprendre l'indémêlable enchevêtrement de passion et d'astreinte, d'intérêt et de désintéressement personnels et familiaux qu'elles impliquent, j'ai braqué pour la dernière période le projecteur sur la famille Caillé. Mais la production de la revue, ainsi qu'à un moindre titre celle des livres, reste l'aboutissement de l'activité d'un réseau amical, comme par le passé - plus encore, sans doute -, même si les personnes impliquées ont en partie changé et si le réseau s'est élargi. Nous avons toujours tenu Serge Latouche et moi à assurer un minimum de trois réunions publiques du MAUSS par an (le samedi matin); première occasion de nous retrouver. Nous nous sommes réunis presque chaque année à 10-15, au début, et à une bonne vingtaine désormais grâce à l'appoint de la jeunesse montante pour les «Trois jours annuels du MAUSS». Seconde occasion de faire le point et de discuter du possible et du souhaitable. Enfin, si Ahmet Insel, qui a longtemps été mobilisé par d'autres tâches (la vice-présidence de l'Université Paris I, et, encore aujourd'hui, l'animation d'une revue et d'une maison d'édition turques, la rédaction d'une chronique dans un grand quotidien également turc ainsi que la contribution à la gestion de l'Université Galatassaray à Istanboul) est moins présent que par le passé, d'autres ont pris le relais. Ahmet est réintervenu sur la question du revenu de citoyenneté avec le $\mathrm{n}^{\circ} 7$, Vers un revenu minimum inconditionnel? JeanLouis Laville a coordonné avec moi le $\mathrm{n}^{\circ} 11$ sur l'Association (Une seule solution, l'association?). Serge Latouche a assuré la préparation du $\mathrm{n}^{\circ} 13$, Le retour de l'ethnocentrisme. Jacques Dewitte a été largement l'âme du n 14 , Villes bonnes à vivre, villes invivables. Réintervention d'Ahmet pour le 15 (et pour le n' 20 qui va sortir d'ici un mois). Première manifestation centrale de Philippe Chanial (et aussi de Bruno Viard) avec l'histoire de la pensée socialiste dans le $\mathrm{n}^{\circ} 16$. La série des trois numéros sur le constructivisme et le naturalisme, les $n^{\text {os }} 17,18$ et 19 , a été entreprise en collaboration entre Philippe Chanial, Frédéric Vandenberghe et moimême. Et, bien sûr ici, je ne parle pas des articles des uns et des autres, qui tous ensemble font le MAUSS et sa réflexion collective.

Au bout du compte, à la suite de ce travail collectif largement informel (et solidaire), nous sommes devenus visibles et lisibles et avons des lecteurs fidèles et passionnés (même s'ils le disent rarement) dans des camps et des chapelles très divers, ce que je trouve fortement gratifiant. En un sens, nous pouvons donc dire que nous avons «réussi ». Au moins à exister, ce qui n’est déjà pas si mal. Est-ce 
au prix d'un abandon et d'une perte d'âme, demanderont peut-être certains? Et, surtout, qui est ce «nous» qui a réussi? Je n'aborderai pas la première question. Mon sentiment est que nous n'avons perdu aucune âme mais que nous avons pris du corps (et de la bouteille). En revanche, malgré la rencontre consacrée il y a quelques années au thème «Qui sommes-nous?», nous avons un peu de mal à répondre à cette question de l'identité du nous et il faut y revenir.

\section{QUI SOMMES-NOUS?}

Qui sont les maussiens ou les Maussiens? Qui est Maussien? Qui ne l'est pas? La seule réponse claire à cette question, pour l'instant, est d'ordre juridique. Jusqu'en 2003, étaient Maussiens les membres de l'association MAUSS qui avaient payé 5'000 FF en vue d'assurer la publication de la revue et de la collection. Soit, nous l'avons vu, 20 cotisants. Vingt Maussiens officiels, donc. Mais cette définition juridique n'est évidemment pas pleinement satisfaisante. Officiellement, chacun aurait dû compléter son don premier par une cotisation annuelle. Or très peu à l'époque l'ont fait régulièrement. Largement par ma faute. Je n'envoyais plus guère, en effet, d'appels de cotisation ou de renouvellement d'abonnement aux Maussiens - mais il faut dire que le taux de réponse était faible... -, d'autant moins incités à s'en acquitter qu'ils recevaient systématiquement toutes nos productions. Mais surtout, un certain nombre des associés ont payé leur cotisation première par sympathie générale pour la liberté intellectuelle plus que par réelle adhésion au programme intellectuel précis du MAUSS (je pense à Henri Denis et Giovanni Busino), d'autres ne viennent guère aux réunions (P. Alphandéry ou P. Bitoun - pourtant champion de ping-pong en titre du MAUSS devant le pourtant presque imbattable Julien Rémy -, qui s'est peu manifesté depuis qu'il habite Strasbourg). D'autres encore ont carrément rompu (B. Guerrien, suite au numéro sur socialisme et totalitarisme, car il ne supportait pas cette catégorie de totalitarisme).

Quant à la communauté d'idées, elle n'est pas si facile à cerner. Le critère le plus tentant, celui que, pour ma part, je mets en œuvre mi-implicitement mi-explicitement, c'est la fidélité à la trajectoire intellectuelle, morale et politique de Marcel Mauss. Mais qui en juge? Et, comme le faisait à juste titre observer récemment Julien Rémy, à ce compte là, Serge Latouche, qui ne parle guère de don (ni même de démocratie) est un maussien incertain (s'il est MAUSSien assuré). A l'inverse, nombre d'auteurs étrangers que nous ne voyons guère ou jamais (Ilana Silber, David Graeber, Chris Gregory, les amis brésiliens ou italiens, etc.) sont à la fois maussiens et au moins de cœur Maussiens. Et Marcel Hénaff, est-il plus ou moins $\mathrm{m}(\mathrm{M})$ aussien que d'autres, a-t-il été demandé à St Jacut? Il veut, semble-til, absolument affirmer sa différence avec nous, mais il croit plus au paradigme du don que Serge. Mais de quel paradigme du don parlons-nous? Agonistique? Forcément et uniquement agonistique? Ou avons-nous en tête une vision un peu plus vaste? Bref, il y a presque autant de cas de figures que de personnes.

A certains égards ce n'est guère gênant, au contraire. Cette diversité vivante a son charme (quand elle ne devient pas hargneuse parce que tel ou tel veut imposer sa vision comme la seule juste). Mais la question reste posée de savoir qui est habilité à parler au nom du MAUSS. Rappel: il y a une dizaine d'années, j'ai eu 
la désagréable surprise d'apprendre par la rédaction du Monde qu'Alain de Benoist se présentait dans le Who is who? comme membre du MAUSS. Interrogé par moi, il m'a répondu qu'il en avait bien le droit puisqu'il avait envoyé une cotisation de $40 \mathrm{FF}$ qui ne lui avait pas été retournée... Un peu plus de formalisme ne nuirait sans doute pas, nous disions-nous donc fin 2002. Au minimum, il y a des arbitrages à effectuer. Le problème ne s'est pas posé souvent, mais il pourrait devenir plus fréquent. Par exemple, il y a 5/6 ans, S. Latouche voulait publier un livre de son ami Michael Singleton. Je n'étais pas d'accord, le trouvant trop abscons et mal ficelé. Serge a suggéré un arbitrage par Philippe Rospabé et AnneMarie Fixot qui ont tranché en mon sens. Je sais aussi qu'il m'en a voulu de lui proposer de placer dans le $\mathrm{n}^{\circ} 13$, dont il était responsable, un article de JeanBaptiste de Foucauld (je ne crois pas l'avoir contraint, contrairement à ce qu'il laisse entendre, mais il est vrai que j'ai insisté et que je ne voulais pas que ce numéro fût intégralement latouchien car je crois les positions de Serge, quelque sympathie que nous ayons tous pour lui, minoritaires au sein du MAUSS). De même, il m'a reproché d'avoir placé un article de Pascal Lamy dans le $\mathrm{n}^{\circ} 20$. La consultation éclair que j'ai lancée sur le mail auprès d'une dizaine de personnes m'a rassuré quant au pluralisme (agonistique, ça va sans dire) et à l'ouverture d'esprit des Maussiens. Mais s'il existait des procédures d'arbitrage plus formelles, ce ne serait pas un mal, assurément.

\section{VOILÀ OÙ NOUS EN ÉTIONS FIN 2002}

Au plan institutionnel, les choses ont passablement évolué depuis. Suite à l'Assemblée générale que la petite histoire que je viens de raconter visait à préparer, il est maintenant possible de devenir membre de l'association du MAUSS avec une cotisation de $100 €$ à condition bien sûr d'être agréé par le bureau du MAUSS, ce qui suppose d'avoir écrit dans la revue et d'y avoir été apprécié. Le MAUSS compte désormais plus de soixante membres, répartis entre Vieux-MAUSSiens (les adhérents primitifs) et Jeunes-MAUSSiens, qui vivent en bonne entente. La liste des noms, souvent prestigieux, qui figurent sur la page de garde de la revue, témoigne des sympathies qu'elle a peu à peu suscitées très audelà du cercle des croyants aux vertus épistémologiques, éthiques et politiques de l'œuvre de Mauss et de ses héritiers proclamés. La Revue explore sans cesse de nouveaux champs. La Bibliothèque du MAUSS s'enrichit régulièrement d'ouvrages nouveaux (à quand un livre de G. Berthoud?). Tout irait donc plutôt bien si les finances et l'appareillage technique du MAUSS n'étaient pas en fait de plus en plus fragiles. Le travail gratuit et la mobilisation permanente ne peuvent pas durer éternellement. Pour ma part, l'âge commençant à venir, je ressens de plus en plus fortement le besoin de réunir les fils de toutes les idées émises, esquissées, brassées dans les dizaines de milliers de pages et le bon millier d'articles que nous avons publiés. Il nous faut à la fois les approfondir et, plus encore peut-être, les exposer de manière plus pédagogique en montrant, notamment aux étudiants et doctorants, comment ce qui leur semble parfois trop abstrait et vaguement impalpable est en fait éminemment concret et aisément opérationnalisable. Pour ce travail d'explicitation, de clarification et de systématisation, je sais que l'apport de Gérald Berthoud, toujours impatient d'aller plus loin et plus profondément, 
sera absolument essentiel maintenant qu'il est libéré de nombre de ses obligations académiques. Et qu'il saura nous aider à franchir une nouvelle étape de l'aventure commune qu'il a impulsée il y a vingt-cinq ans. Merci Gérald.

Département de sociologie

Université de Paris X - Nanterre

Mauss1981@aol.com 